\title{
On fractional derivatives with generalized Mittag-Leffler kernels
}

\author{
Thabet Abdeljawad ${ }^{1}$ and Dumitru Baleanu $u^{2,3^{*}}$
}

\author{
"Correspondence: \\ dumitru@cankaya.edu.tr \\ ${ }^{2}$ Department of Mathematics, \\ Cankaya University, Ankara, Turkey \\ ${ }^{3}$ Institute of Space Sciences, \\ Magurele-Bucharest, Romania \\ Full list of author information is \\ available at the end of the article
}

\begin{abstract}
Fractional derivatives with three parameter generalized Mittag-Leffler kernels and their properties are studied. The corresponding integral operators are obtained with the help of Laplace transforms. The action of the presented fractional integrals on the Caputo and Riemann type derivatives with three parameter Mittag-Leffler kernels is analyzed. Integration by parts formulas in the sense of Riemann and Caputo are proved and then used to formulate the fractional Euler-Lagrange equations with an illustrative example. Certain nonconstant functions whose fractional derivatives are zero are determined as well.

Keywords: Fractional derivatives with generalized Mittag-Leffler kernels; Generalized Mittag-Leffler function; Laplace transform convolution; Euler-Lagrange equation; Integration by parts
\end{abstract}

\section{Basics and preliminaries}

Fractional calculus is an emerging field with several real world applications in various areas of science and engineering [1-13].

Recently, generalized versions of the standard classical fractional derivatives and their discrete versions (see, for example, Refs. [14-19]) are required by a better description of the dynamics of so-called complex systems. The extension of these fractional operators with nonsingular kernels and the Lyapunov type inequalities and their discrete versions have been later investigated in [20-23]. Further, the monotonicity analysis of such discrete fractional operators has been also studied in $[24,25]$. Thus, we have to investigate if the new fractional derivative with Mittag-Leffler kernel can be used properly for treating the control problems and if it can be used successfully to produce new fractional EulerLagrange equations. As it can be seen, these types of equations contain two types of derivatives, the left and right ones, and they are new types of equations. The solutions of these types of equations were used successfully to treat several complex oscillatory systems [2633]. In fact, the fractional variational principles provided the scientific community with a new type of Euler-Lagrange equations which opened new directions in the field of numerical analysis.

Keeping the above mentioned things in our minds, we discuss in our manuscript the fractional operators with three parameter generalized Mittag-Leffler kernels and we present the integration by parts formulas, and finally we obtain the related fractional Euler-Lagrange equations. Therefore, we generalize the results obtained in [15, 17]. The

(c) The Author(s) 2018. This article is distributed under the terms of the Creative Commons Attribution 4.0 International License (http://creativecommons.org/licenses/by/4.0/), which permits unrestricted use, distribution, and reproduction in any medium, provided you give appropriate credit to the original author(s) and the source, provide a link to the Creative Commons license, and indicate if changes were made. 
defined fractional derivatives depend on a limiting approach of weighted versions of Prabhakar fractional integrals [34] with the help of delta Dirac functions. Prabhakar fractional integrals were investigated in [35] as well.

We recall that the $Q$-operator action, $(Q f)(t)=f(a+b-t)$, was utilized earlier in fractional calculus [1-4] to relate left and right type fractional integrals and derivatives. Then, recently, it has been employed to relate left and right fractional sums and differences [36, 37]. In our article, we will use the action of $Q$-operator in order to define and to confirm our definitions of fractional derivatives with Mittag-Leffler function kernels.

Definition 1 The Mittag-Leffler function of one parameter is written as

$$
E_{\alpha}(\lambda, z)=E_{\alpha}\left(\lambda z^{\alpha}\right)=\sum_{k=0}^{\infty} \lambda^{k} \frac{z^{\alpha k}}{\Gamma(\alpha k+1)}, \quad(0 \neq \lambda \in \mathbb{R}, z \in \mathbb{C} ; \operatorname{Re}(\alpha)>0),
$$

and the expression of the one with two parameters $\alpha$ and $\beta$ has the following form:

$$
E_{\alpha, \beta}(\lambda, z)=z^{\beta-1} E_{\alpha, \beta}\left(\lambda z^{\alpha}\right)=\sum_{k=0}^{\infty} \lambda^{k} \frac{z^{\alpha k+\beta-1}}{\Gamma(\alpha k+\beta)}, \quad(0 \neq \lambda \in \mathbb{R}, z, \beta \in \mathbb{C} ; \operatorname{Re}(\alpha)>0),
$$

where $E_{\alpha, 1}(\lambda, z)=E_{\alpha}(\lambda, z)$.

We recall that the formula of the generalized ML of three parameters is

$$
E_{\alpha, \beta}^{\sigma}(z)=\sum_{k=0}^{\infty}(\sigma)_{k} \frac{z^{k}}{k ! \Gamma(\alpha k+\beta)}
$$

where $(\sigma)_{k}=\sigma(\sigma+1) \cdots(\sigma+k-1)$ is the Pochhammer symbol. Notice that $(1)_{k}=k$ ! so that $E_{\alpha, \beta}^{1}(z)=E_{\alpha, \beta}(z)$.

Using a modified version, the following is the ML function of three parameters:

$$
E_{\alpha, \beta}^{\sigma}(\lambda, z)=\sum_{k=0}^{\infty} \lambda^{k}(\sigma)_{k} \frac{z^{\alpha k+\beta-1}}{k ! \Gamma(\alpha k+\beta)}
$$

Definition 2 Let $s \in \mathbb{R}$ and $f_{1}, g_{1}:[a, \infty) \rightarrow \mathbb{R}$ be functions. The convolution of $f_{1}$ with $g_{1}$ starting from $a$ is written as

$$
\left(f_{1} * g_{1}\right)(t)=\int_{a}^{t} g_{1}(t-s+a) f_{1}(s) d s
$$

and the Laplace transform starting from $a$ of $f_{1}$ as

$$
\left\{\mathbb{L}_{a} f_{1}(t)\right\}(z)=\int_{a}^{\infty} f_{1}(t) e^{-z(t-a)} d t
$$

When $a=0$, we use $\mathbb{L}$ instead of $\mathbb{L}_{a}$.

Depending on Definition 2, we can prove the following $a$-convolution Laplace identity that we shall use throughout this article. 
Proposition 1 For $f$, $g$ defined on $[a, \infty)$ we conclude that

$$
\left(\mathbb{L}_{a}(f * g)\right)(z)=\left(\mathbb{L}_{a} f\right)(z)\left(\mathbb{L}_{a} g\right)(z) .
$$

Proof The proof is straightforward and can be achieved by using definition and interchanging the orders of the integrals.

\section{Generalized fractional derivatives with singular ML kernels with three parameters}

Definition 3 The generalized $\mathrm{ABR}$ and $\mathrm{ABC}$ fractional derivatives with kernel $E_{\alpha, \mu}^{\gamma}(\lambda, t)$, where $0<\alpha<1, \operatorname{Re}(\mu)>0, \gamma \in \mathbb{R}$, and $\lambda=\frac{-\alpha}{1-\alpha}$, are defined respectively by

$$
\begin{aligned}
\left({ }_{a}^{\mathrm{ABC}} D^{\alpha, \mu, \gamma} f\right)(x) & =\frac{B(\alpha)}{1-\alpha} \int_{a}^{x} E_{\alpha, \mu}^{\gamma}(\lambda, x-t) f^{\prime}(t) d t \\
& =\frac{B(\alpha)}{1-\alpha} E_{\alpha, \mu}^{\gamma}(\lambda, x-a) * f^{\prime}(x) .
\end{aligned}
$$

The right one by

$$
\left({ }^{\mathrm{ABC}} D_{b}^{\alpha, \mu, \gamma} f\right)(x)=\frac{-B(\alpha)}{1-\alpha} \int_{x}^{b} E_{\alpha, \mu}^{\gamma}(\lambda, t-x) f^{\prime}(t) d t
$$

and

$$
\begin{aligned}
\left({ }_{a}^{\mathrm{ABR}} D^{\alpha, \mu, \gamma} f\right)(x) & =\frac{B(\alpha)}{1-\alpha} \frac{d}{d x} \int_{a}^{x} E_{\alpha, \mu}^{\gamma}(\lambda, x-t) f(t) d t \\
& =\frac{B(\alpha)}{1-\alpha} \frac{d}{d x} E_{\alpha, \mu}^{\gamma}(\lambda, x-a) * f(x) .
\end{aligned}
$$

The right one by

$$
\left({ }^{\mathrm{ABR}} D_{b}^{\alpha, \mu, \gamma} f\right)(x)=\frac{-B(\alpha)}{1-\alpha} \frac{d}{d x} \int_{x}^{b} E_{\alpha, \mu}^{\gamma}(\lambda, t-x) f(t) d t .
$$

Here $B(0)=B(1)=1$. Simply $B(\alpha)$ can be chosen as 1 .

Remark 1 Note that the above generalized type fractional derivatives have singular kernels for $0<\mu<1$. However, the one parameter ML function kernel defined in [15] is nonsingular. Also, the limiting process $\alpha, \mu, \gamma \rightarrow 1$ gives the ordinary derivative.

Theorem 1 ([35]) For $\rho, \mu, \gamma, v, \sigma, \lambda \in \mathbb{C}(\operatorname{Re}(\rho), \operatorname{Re}(\mu), \operatorname{Re}(v)>0)$, we have

$$
\int_{0}^{x}(x-t)^{\mu-1} E_{\rho, \mu}^{\gamma}\left(\lambda[x-t]^{\rho}\right) t^{\nu-1} E_{\rho, \nu}^{\sigma}\left(\lambda t^{\rho}\right) d t=x^{\mu+\nu-1} E_{\rho, \mu+\nu}^{\gamma+\sigma}\left(\lambda x^{\rho}\right) .
$$

Particularly, if $\gamma=1, \mu=1$, and $\rho=\alpha$, we have

$$
\int_{0}^{x} E_{\alpha}\left(\lambda[x-t]^{\alpha}\right) t^{\nu-1} E_{\alpha, v}^{\sigma}\left(\lambda t^{\alpha}\right) d t=x^{\nu} E_{\alpha, 1+v}^{1+\sigma}\left(\lambda x^{\alpha}\right) .
$$


Remark 2 If we utilize the modified notation of ML, then (11) becomes

$$
\int_{0}^{x} E_{\rho, \mu}^{\gamma}(\lambda, x-t) E_{\rho, \nu}^{\sigma}(\lambda, t) d t=E_{\rho, \mu+\nu}^{\gamma+\sigma}(\lambda, x),
$$

and (12) is written as

$$
\int_{0}^{x} E_{\alpha}(\lambda, x-t) E_{\alpha, v}^{\sigma}(\lambda, t) d t=E_{\alpha, 1+v}^{1+\sigma}(\lambda, x) .
$$

For $\alpha, \mu, \gamma, \lambda \in \mathbb{C}(\operatorname{Re}(\alpha)>0)$, and $n \in \mathbb{N}$, we conclude that [3]

$$
\left(\frac{d}{d z}\right)^{n}\left[E_{\alpha, \mu}^{\gamma}(\lambda, z)\right]=E_{\alpha, \mu-n}^{\gamma}(\lambda, z) .
$$

Now, from (13) and (15), we see that

$$
{ }_{0}^{\mathrm{ABR}} D^{\alpha, \mu, \gamma}\left[E_{\alpha, \nu}^{\sigma}(\lambda, x)\right]=\frac{B(\alpha)}{1-\alpha} \frac{d}{d x}\left[E_{\alpha, \mu+\nu}^{\gamma+\sigma}(\lambda, x)\right]=\frac{B(\alpha)}{1-\alpha} E_{\alpha, \nu+\mu-1}^{\gamma+\sigma}(\lambda, x),
$$

and

$$
\begin{aligned}
{ }_{0}^{\mathrm{ABC}} D^{\alpha, \mu, \gamma}\left[E_{\alpha, \nu}^{\sigma}(\lambda, x)\right] & =\frac{B(\alpha)}{1-\alpha} \int_{0}^{x} E_{\alpha, \mu}^{\gamma}(\lambda, x-t) \frac{d}{d t}\left[E_{\alpha, v}^{\sigma}(\lambda, t)\right] d t \\
& =\frac{B(\alpha)}{1-\alpha} E_{\alpha, v+\mu-1}^{\gamma+\sigma}(\lambda, x) .
\end{aligned}
$$

Remark 3 Noting that

$$
E_{\alpha, v+\mu-1}^{0}(\lambda, x)=\frac{x^{\nu+\mu-2}}{\Gamma(\nu+\mu-1)} \rightarrow 0, \quad v \rightarrow 1-\mu, 0<\mu \leq 1,
$$

from (16) and (17) for $\sigma=-\gamma$, it implies that

$$
G_{\gamma}(x)=\frac{1-\alpha}{B(\alpha)} E_{\alpha, \nu}^{-\gamma}(\lambda, x), \quad \nu \rightarrow 1-\mu
$$

is a nonzero function such that its $\mathrm{ABR}$ and $\mathrm{ABC}$ derivatives are zero. By inspection we report that the function $G(x)$ tends to 1 as $\mu \rightarrow 1^{-}$and $\alpha \rightarrow 1$ with $\gamma=1$. Also, it is of interest to study the fractional polynomial function $G_{\gamma}(x)$ with $\gamma=1,2,3, \ldots$ (see Example 1 below).

Now, we solve the equation $\left({ }_{a}^{\mathrm{ABR}} D^{\alpha, \mu, \gamma} f\right)(t)=u(t)$ with $\gamma=1$ to find the fractional integral operator of two parameters. After we perform the Laplace transform to both sides, we utilize the convolution identity in Proposition 1 and make use of the fact that

$$
\left(\mathbb{L}_{a} E_{\alpha, \beta}^{\gamma}(\lambda, t-a)\right)(s)=s^{-\beta}\left[1-\lambda s^{-\alpha}\right]^{-\gamma},
$$

and that $f(t)$ is continuous at $a$ to have

$$
\left(\mathbb{L}_{a_{a}}^{\mathrm{ABR}} D^{\alpha, \mu, 1} f(t)\right)(s)=\frac{B(\alpha)}{1-\alpha}\left(\mathbb{L}_{a} \frac{d}{d t}\left[f(t) * E_{\alpha, \mu}(\lambda, t-a)\right]\right)(s)
$$




$$
\begin{aligned}
& =\frac{B(\alpha)}{1-\alpha} S \cdot s^{-\mu}\left[1-\lambda s^{-\alpha}\right]^{-1} F(s)-0 \\
& =U(s),
\end{aligned}
$$

where $U(s)=\left(\mathbb{L}_{a} u(t)\right)(s), F(s)=\left(\mathbb{L}_{a} f(t)\right)(s)$, and $\lambda=\frac{-\alpha}{1-\alpha}$. From which it follows that

$$
F(s)=\frac{1-\alpha}{B(\alpha)} s^{\mu-1}\left[1-\lambda s^{-\alpha}\right] U(s)=s^{\mu-1}\left[\frac{1-\alpha}{B(\alpha)}+\frac{\alpha}{B(\alpha)} s^{-\alpha}\right] U(s) .
$$

Utilizing the inverse Laplace, we see that

$$
f(t)=\frac{1-\alpha}{B(\alpha)}\left({ }_{a} I^{1-\mu} u\right)(t)+\frac{\alpha}{B(\alpha)}\left({ }_{a} I^{1-\mu_{+} \alpha} u\right)(t)
$$

As a result, we have the following definition.

Definition 4 Let $f$ be a continuous function defined on an interval $[a, b]$ and assume $0<$ $\alpha \leq 1, \mu>0$. Then the left and right fractional integrals of two parameters $\alpha$ and $\mu$ are defined respectively by

$$
\left({ }_{a}^{\mathrm{AB}} I^{\alpha, \mu} u\right)(t)=\frac{1-\alpha}{B(\alpha)}\left({ }_{a} I^{1-\mu} u\right)(t)+\frac{\alpha}{B(\alpha)}\left({ }_{a} I^{1-\mu_{+} \alpha} u\right)(t)
$$

and

$$
\left({ }^{\mathrm{AB}} I_{b}^{\alpha, \mu} u\right)(t)=\frac{1-\alpha}{B(\alpha)}\left(I_{b}^{1-\mu} u\right)(t)+\frac{\alpha}{B(\alpha)}\left(I_{b}^{1-\mu_{+} \alpha} u\right)(t)
$$

where $\left({ }_{a} I^{\alpha} u\right)(t)$ and $\left(I_{b}^{\alpha} u\right)(t)$ are the left and right Riemann fractional integrals.

Remark 4 Note that if $\mu$ tends to 1 in Definition 4, we have $\left.{ }_{a}^{\mathrm{AB}} I^{\alpha, 1} u\right)(t)=\left({ }_{a}^{\mathrm{AB}} I^{\alpha} u\right)(t)$ and $\left({ }^{\mathrm{AB}} I_{b}^{\alpha, 1} u\right)(t)=\left({ }^{\mathrm{AB}} I_{b}^{\alpha} u\right)(t)$. The case of finding explicit formulas for the left and right $\mathrm{AB}$ fractional integrals of order $\alpha, \mu, \gamma$ when $\gamma \neq 1$ has not been treated above. However, it is possible to formulate the particular cases $\gamma=2,3,4, \ldots$ with the help of Laplace transforms. In fact, the $\mathrm{AB}$ fractional integrals of order $0<\alpha \leq 1, \mu>0, \gamma=1,2, \ldots$, are given by

$$
\left({ }_{a}^{\mathrm{AB}} I^{\alpha, \mu, \gamma} u\right)(t)=\sum_{i=0}^{\gamma}\left(\begin{array}{c}
\gamma \\
i
\end{array}\right) \frac{\alpha^{i}}{B(\alpha)(1-\alpha)^{i-1}}\left({ }_{a} I^{\alpha i+1-\mu} u\right)(t)
$$

and

$$
\left({ }^{\mathrm{AB}} I_{b}^{\alpha, \mu, \gamma} u\right)(t)=\sum_{i=0}^{\gamma}\left(\begin{array}{c}
\gamma \\
i
\end{array}\right) \frac{\alpha^{i}}{B(\alpha)(1-\alpha)^{i-1}}\left(I_{b}^{\alpha i+1-\mu} u\right)(t) .
$$

From [15] we recall the following:

$$
\left({ }_{0}^{\mathrm{ABC}} D^{\alpha} f\right)(t)=\left({ }_{0}^{\mathrm{ABR}} D^{\alpha} f\right)(t)-\frac{B(\alpha)}{1-\alpha} f(0) E_{\alpha}(\lambda, t) .
$$


Actually, the $a$-version is $(f$ is regular at $a)$

$$
\left({ }_{a}^{\mathrm{ABC}} D^{\alpha} f\right)(t)=\left({ }_{a}^{\mathrm{ABR}} D^{\alpha} f\right)(t)-\frac{B(\alpha)}{1-\alpha} f(a) E_{\alpha}(\lambda, t-a)
$$

and the $b$-right fractional version is

$$
\left({ }^{\mathrm{ABC}} D_{b}^{\alpha} f\right)(t)=\left({ }^{\mathrm{ABR}} D_{b}^{\alpha} f\right)(t)-\frac{B(\alpha)}{1-\alpha} f(b) E_{\alpha}(\lambda, b-t) .
$$

More generally, we can state and give proof of the following.

Theorem 2 (The relation between the generalized ABR and the generalized ABC fractional derivatives) For any $0<\alpha<1, \mu>0, \gamma \in \mathbb{R}$, and $f$ is regular at a, we have

- $\left({ }_{a}^{\mathrm{ABC}} D^{\alpha, \mu, \gamma} f\right)(x)=\left({ }_{a}^{\mathrm{ABR}} D^{\alpha, \mu, \gamma} f\right)(x)-\frac{B(\alpha)}{1-\alpha} f(a) E_{\alpha, \mu}^{\gamma}(\lambda, x-a)$,

- $\left({ }^{\mathrm{ABC}} D_{b}^{\alpha, \mu, \gamma} f\right)(x)=\left({ }^{\mathrm{ABR}} D_{b}^{\alpha} f\right)(x)-\frac{B(\alpha)}{1-\alpha} f(b) E_{\alpha, \mu}^{\gamma}(\lambda, b-x)$,

where always $\lambda=\frac{-\alpha}{1-\alpha}$.

Proof From the relations

$$
\mathbb{L}_{a}\left\{\left({ }_{a}^{\mathrm{ABR}} D^{\alpha, \mu, \gamma} f\right)(t)\right\}(s)=\frac{B(\alpha)}{1-\alpha} s^{1-\mu} F(s)\left[1-\lambda s^{-\alpha}\right]^{-\gamma}
$$

and

$$
\mathbb{L}_{a}\left\{\left(\begin{array}{l}
\mathrm{ABC} \\
a
\end{array} D^{\alpha, \mu, \gamma} f\right)(t)\right\}(s)=\frac{B(\alpha)}{1-\alpha} s^{1-\mu} F(s)\left[1-\lambda s^{-\alpha}\right]^{-\gamma}-\frac{B(\alpha)}{1-\alpha} f(a) s^{-\mu}\left[1-\lambda s^{-\alpha}\right]^{-\gamma},
$$

we conclude that

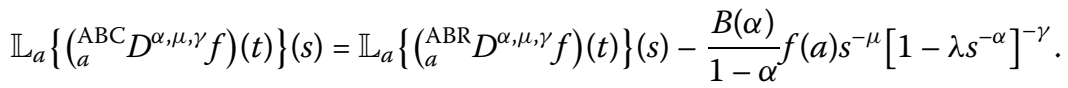

Applying the inverse Laplace to (28), we finish our conclusion in the first part. The second part can be proved with the help of the first part as well as the $Q$-operator action.

Using Theorem 2, (19), (20), and the identity (see [3] page 78 or Theorem 3 in [35])

$$
\left({ }_{a} I^{\alpha}(t-a)^{\beta-1} E_{\mu, \beta}^{\gamma}\left[\lambda(t-a)^{\mu}\right](x)=(x-a)^{\alpha+\beta-1} E_{\mu, \alpha+\beta}^{\gamma}\left[\lambda(x-a)^{\mu}\right],\right.
$$

or in a modified version as

$$
\left({ }_{a} I^{\alpha} E_{\mu, \beta}^{\gamma}(\lambda, t-a)(x)=E_{\mu, \alpha+\beta}^{\gamma}(\lambda, x-a),\right.
$$

we can conclude the following.

Proposition 2 For $0<\alpha<1, \mu>0, \gamma=1$, we have

$$
\begin{aligned}
\left({ }_{a}^{\mathrm{AB}} I_{a}^{\alpha, \mu, 1 \mathrm{ABC}} D^{\alpha, \mu, 1} f\right)(x) & =f(x)-f(a) E_{\alpha, 1}(\lambda, x-a)-\frac{\alpha}{1-\alpha} f(a) E_{\alpha, \alpha+1}(\lambda, x-a) \\
& =f(x)-f(a) .
\end{aligned}
$$


Similarly,

$$
\left({ }^{\mathrm{AB}} I_{b}^{\alpha, \mu \mathrm{ABC}} D_{b}^{\alpha, \mu} f\right)(x)=f(x)-f(b) .
$$

In the proof of Proposition 2, we need to make use of the identity

$$
E_{\alpha, 1}(\lambda, x-a)-\lambda E_{\alpha, \alpha+1}(\lambda, x-a)=1 .
$$

Remark 5 From Remark 4 with $\gamma=2$, for $0<\alpha<1, \mu>0$, we have

$$
\begin{aligned}
\left({ }_{a}^{\mathrm{AB}} I^{\alpha, \mu, 2} u\right)(t)= & \frac{1-\alpha}{B(\alpha)}\left({ }_{a} I^{1-\mu} u\right)(t)+\frac{2 \alpha}{B(\alpha)}\left({ }_{a} I^{\alpha-\mu+1} u\right)(t) \\
& +\frac{\alpha^{2}}{B(\alpha)(1-\alpha)}\left({ }_{a} I^{2 \alpha-\mu+1} u\right)(t)
\end{aligned}
$$

In particular,

$$
\begin{aligned}
\left({ }_{a}^{\mathrm{AB}} I^{\alpha, 1,2} u\right)(t)= & \frac{1-\alpha}{B(\alpha)} u(t)+\frac{2 \alpha}{B(\alpha)}\left({ }_{a} I^{\alpha} u\right)(t) \\
& +\frac{\alpha^{2}}{B(\alpha)(1-\alpha)}\left({ }_{a} I^{2 \alpha} u\right)(t) .
\end{aligned}
$$

Hence, we can generalize Proposition 2 for other values of $\gamma$ as follows.

Proposition 3 For $0<\alpha<1, \mu>0, \gamma=2$, and $\lambda=\frac{-\alpha}{1-\alpha}$, we have

$$
\begin{aligned}
&{\left.\underset{a}{\mathrm{AB}} I_{a}^{\alpha, \mu, 2 \mathrm{ABC}} D^{\alpha, \mu, 2} f\right)(x)=}(x)-f(a)\left[E_{\alpha, 1}^{2}(\lambda, x-a)-2 \lambda E_{\alpha, \alpha+1}^{2}(\lambda, x-a)\right. \\
&+\lambda^{2} E_{\alpha, 2 \alpha+1}^{2}(\lambda, x-a) \\
&= f(x)-f(a) \\
& \times[1+\sum_{k=2}^{\infty} \lambda^{k} \frac{(x-a)^{\alpha k}}{\Gamma(\alpha k+1)} \underbrace{\left.\left(\frac{(2)_{k}}{k !}-\frac{2(2)_{k-1}}{(k-1) !}+\frac{(2)_{k-2}}{(k-2) !}\right)\right]}_{=0} \\
&= f(x)-f(a) .
\end{aligned}
$$

Similarly, by the action of the Q-operator, we have

$$
\left({ }^{\mathrm{AB}} I_{b}^{\alpha, \mu, 2 \mathrm{ABC}} D_{b}^{\alpha, \mu, 2} f\right)(x)=f(x)-f(b) .
$$

More generally, if we proceed inductively on $\gamma \in \mathbb{N}$ by making use of the identity

$$
\sum_{i=0}^{\gamma}(-1)^{i}\left(\begin{array}{l}
\gamma \\
i
\end{array}\right) \frac{(\gamma)_{k-i}}{(k-i) !}=0, \quad \forall k \geq \gamma
$$

we can state the following $\gamma$-version of Proposition 2 and Proposition 3. 
Theorem 3 For $0<\alpha<1, \mu>0, \gamma \in \mathbb{N}$, and $\lambda=\frac{-\alpha}{1-\alpha}$, we have

$$
\begin{aligned}
\left({ }_{a}^{\mathrm{AB}} I_{a}^{\alpha, \mu, \gamma} \underset{a}{\mathrm{ABC}} D^{\alpha, \mu, \gamma} f\right)(x) & =f(x)-f(a) \sum_{k=0}^{\gamma}(-1)^{k} \lambda^{k} E_{\alpha, \alpha k+1}^{\gamma}(\lambda, x-a) \\
& =f(x)-f(a) .
\end{aligned}
$$

Similarly, by using Q-operator, we get

$$
\begin{aligned}
\left({ }^{\mathrm{AB}} I_{b}^{\alpha, \mu, \gamma \mathrm{ABC}} D_{b}^{\alpha, \mu, \gamma} f\right)(x) & =f(x)-f(b) \sum_{k=0}^{\gamma}(-1)^{k} \lambda^{k} E_{\alpha, \alpha k+1}^{\gamma}(\lambda, b-x) \\
& =f(x)-f(b) .
\end{aligned}
$$

\section{Integration by parts}

Below we shall deal with integration by parts for fractional integrals $\left({ }_{a}^{\mathrm{AB}} I^{\alpha, \mu, \gamma} u\right)(t)$ and derivatives $\left({ }_{a}^{\mathrm{AB}} D^{\alpha, \mu, \gamma} u\right)(t)$ of orders $0<\alpha<1, \operatorname{Re}(\mu)>0, \gamma=1,2,3, \ldots$. The extension for arbitrary $\gamma$ is still open since there is no explicit formula for the integral operator in case $\gamma \notin \mathbb{N}$.

Below we discuss the following function spaces: For $p \geq 1$ and $0<\alpha<1, \mu>0, \gamma \in \mathbb{C}$, we define

$$
\left({ }_{a}^{\mathrm{AB}} I^{\alpha, \mu, \gamma}\right)\left(L_{p}\right)=\left\{f: f={ }_{a}^{\mathrm{AB}} I^{\alpha, \mu, \gamma} \varphi, \varphi \in L_{p}(a, b)\right\}
$$

and

$$
\left({ }^{\mathrm{AB}} I_{b}^{\alpha, \mu, \gamma}\right)\left(L_{p}\right)=\left\{f: f={ }^{\mathrm{AB}} I_{b}^{\alpha, \mu, \gamma} \phi, \phi \in L_{p}(a, b)\right\} .
$$

The discussion in the previous section shows that

$$
\left({ }_{a}^{\mathrm{AB}} D_{a}^{\alpha, \mu, \gamma} \underset{a \mathrm{AB}}{\alpha, \mu, \gamma} u\right)(t)=u(t)
$$

and

$$
\left({ }^{\mathrm{AB}} D_{b}^{\alpha, \mu, \gamma \mathrm{AB}} I_{b}^{\alpha, \mu, \gamma} u\right)(t)=u(t)
$$

We next prove that $\left({ }_{a}^{\mathrm{AB}} I^{\alpha, \mu, \gamma} \underset{a}{\mathrm{ABR}} D^{\alpha, \mu, \gamma} f\right)(t)=f(t)$ and

$$
\left({ }^{\mathrm{AB}} I_{b}^{\alpha, \mu, \gamma \mathrm{ABR}} D_{b}^{\alpha, \mu, \gamma} f\right)(t)=f(t),
$$

thus the function spaces $\left({ }_{a}^{\mathrm{AB}} I^{\alpha, \mu, \gamma}\right)\left(L_{p}\right)$ and $\left({ }^{\mathrm{AB}} I_{b}^{\alpha, \mu, \gamma}\right)\left(L_{p}\right)$ are nonempty.

Theorem 4 Let $0<\alpha<1, \mu>0, \gamma \in \mathbb{N}$, then the functions $\left({ }_{a}^{\mathrm{ABR}} D^{\alpha, \mu, \gamma} f\right)(t)$ and $\left({ }^{\mathrm{ABR}} D_{b}^{\alpha, \mu, \gamma} f\right)(t)$ satisfy the equations

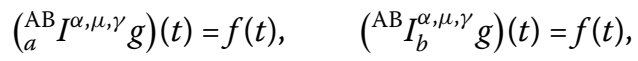

respectively. 
Proof Below we discuss the proof for the left case only. Using the Q-operator, the right case can be proved easily. With the help of the definition we show that the first equation is equivalent to

$$
\sum_{k=0}^{\gamma}\left(\begin{array}{l}
\gamma \\
k
\end{array}\right) \frac{\alpha^{k}}{(1-\alpha)^{k-1} B(\alpha)}\left({ }_{a}^{\alpha k-\mu+1} g\right)(t)=f(t)
$$

For $\gamma=1$, the Laplace transform gives us the following:

$$
s^{\mu-1}\left[\frac{1-\alpha}{B(\alpha)}+\frac{\alpha}{B(\alpha)} s^{-\alpha}\right] G(s)=F(s),
$$

from which it follows that

$$
G(s)=\frac{B(\alpha) F(s)}{s^{\mu-1}\left(1-\alpha+\alpha s^{-\alpha}\right)}=\left(\mathbb{L}_{a_{a}}^{\mathrm{ABR}} D^{\alpha, \mu \cdot 1} f\right)(s) .
$$

After that, the Laplace inverse implies that $g(t)=\left({ }_{a}^{\mathrm{ABR}} D^{\alpha, \mu \cdot 1} f\right)(t)$.

For $\gamma=2$, the Laplace transform gives

$$
\left[1+\frac{2 \alpha s^{-\alpha}}{1-\alpha}+\frac{\alpha^{2}}{(1-\alpha)^{2}} s^{-2 \alpha}\right] G(s)=s^{1-\mu} \frac{B(\alpha)}{1-\alpha} F(s),
$$

from which it follows by (26) with $\gamma=2$ that

$$
G(s)=\frac{B(\alpha) F(s)}{s^{\mu-1}(1-\alpha)\left(1-2 \lambda s^{-\alpha}+\lambda^{2} s^{-2 \alpha}\right)}=\left(\mathbb{L}_{a_{a}}^{\mathrm{ABR}} D^{\alpha, \mu .2} f\right)(s) .
$$

Now, the Laplace inverse gives $g(t)=\left({ }_{a}^{\mathrm{ABR}} D^{\alpha, \mu .2} f\right)(t)$. If we proceed inductively on $\gamma$ by making use of (26) and (21), we conclude that $g(t)=\left({ }_{a}^{\mathrm{ABR}} D^{\alpha, \mu \cdot \gamma} f\right)(t)$. The right case follows by the left case and the action of the $Q$-operator.

Theorem 5 Let $0<\alpha<1, \mu>0, \gamma \in \mathbb{N}, p \geq 1, q \geq 1$, and $\frac{1}{p}+\frac{1}{q} \leq 1+\alpha(p \neq 1$ and $q \neq 1$ in the case $\left.\frac{1}{p}+\frac{1}{q}=1+\alpha\right)$. Then

- If $\varphi(x) \in L_{p}(a, b)$ and $\psi(x) \in L_{q}(a, b)$, then

$$
\int_{a}^{b} \varphi(x)\left({ }_{a}^{\mathrm{AB}} I^{\alpha, \mu, \gamma} \psi\right)(x) d x=\int_{a}^{b} \psi(x)\left({ }^{\mathrm{AB}} I_{b}^{\alpha, \mu, \gamma} \varphi\right)(x) d x
$$

and similarly,

$$
\int_{a}^{b} \varphi(x)\left({ }^{\mathrm{AB}} I_{b}^{\alpha, \mu, \gamma} \psi\right)(x) d x=\int_{a}^{b} \psi(x)\left({ }_{a}^{\mathrm{AB}} I^{\alpha, \mu, \gamma} \varphi\right)(x) d x
$$

- If $f(x) \in{ }^{\mathrm{AB}} I_{b}^{\alpha, \mu, \gamma}\left(L_{p}\right)$ and $g(x) \in{ }_{a}^{\mathrm{AB}} I^{\alpha, \mu, \gamma}\left(L_{q}\right)$, then

$$
\int_{a}^{b} f(x)\left({ }_{a}^{\mathrm{ABR}} D^{\alpha, \mu, \gamma} g\right)(x) d x=\int_{a}^{b}\left({ }^{\mathrm{ABR}} D_{b}^{\alpha, \mu, \gamma} f\right)(x) g(x) d x
$$


and similarly,

$$
\int_{a}^{b} f(x)\left({ }^{\mathrm{AB}} D_{b}^{\alpha, \mu, \gamma} g\right)(x) d x=\int_{a}^{b} g(x)\left({ }_{a}^{\mathrm{AB}} D^{\alpha, \mu, \gamma} f\right)(x) d x
$$

for $f(x) \in{ }_{a}^{\mathrm{AB}} I^{\alpha, \mu, \gamma}\left(L_{p}\right)$ and $g(x) \in{ }^{\mathrm{AB}} I_{b}^{\alpha, \mu, \gamma}\left(L_{q}\right)$.

\section{Proof}

- The proof follows by (21), (22) and the integration by parts for (classical)

Riemann-Liouville fractional integrals.

- From the assumption and the first part, we have

$$
\begin{aligned}
\int_{a}^{b} f(x)\left({ }_{a}^{\mathrm{ABR}} D^{\alpha, \mu, \gamma} g\right)(x) d x & =\int_{a}^{b}\left({ }^{\mathrm{AB}} I_{b}^{\alpha, \mu, \gamma} \phi\right)(x) \cdot\left({ }_{a}^{\mathrm{ABR}} D_{a}^{\alpha, \mu, \gamma}{ }_{a}^{\mathrm{ABR}} I^{\alpha, \mu, \gamma} \varphi\right)(x) d x \\
& =\int_{a}^{b}\left({ }^{\mathrm{AB}} I_{b}^{\alpha, \mu, \gamma} \phi\right)(x) \cdot \varphi(x) d x \\
& =\int_{a}^{b} \phi(x) \cdot\left({ }_{a}^{\mathrm{AB}} I^{\alpha, \mu, \gamma} \varphi\right)(x) d x \\
& =\int_{a}^{b}\left({ }^{\mathrm{ABR}} D_{b}^{\alpha, \mu, \gamma} f\right)(x) g(x) d x
\end{aligned}
$$

The fact that the fractional integral operator and the differential operator are inverses to each other has been used (notice Theorem 4 and above it).

From [35] recall the (left) generalized fractional integral operator

$$
\begin{aligned}
\left(\mathbf{E}_{\rho, \mu, \omega, a^{+}}^{\gamma} \varphi\right)(x) & =\int_{a}^{x}(x-t)^{\mu-1} E_{\rho, \mu}^{\gamma}\left[\omega(x-t)^{\rho}\right] \varphi(t) d t, \quad x>a \\
& =\int_{a}^{x} E_{\rho, \mu}^{\gamma}(\omega, x-t) \varphi(t) d t, \quad x>a .
\end{aligned}
$$

Thus, we define the (right) generalized fractional integral operator as

$$
\begin{aligned}
\left(\mathbf{E}_{\rho, \mu, \omega, b^{-}}^{\gamma} \varphi\right)(x) & =\int_{x}^{b}(t-x)^{\mu-1} E_{\rho, \mu}^{\gamma}\left[\omega(t-x)^{\rho}\right] \varphi(t) d t, \quad x<b \\
& \left.=\int_{x}^{b} E_{\rho, \mu}^{\gamma}(\omega, t-x)\right) \varphi(t) d t, \quad x<b
\end{aligned}
$$

where $E_{\rho, \mu}^{\gamma}(z)=\sum_{k=0}^{\infty} \frac{(\gamma)_{k} z^{k}}{\Gamma(\rho k+\mu) k !}$ is the generalized Mittag-Leffler function which is defined for complex $\rho, \mu, \gamma(\operatorname{Re}(\rho)>0)[3,35]$.

Using notations (45) and (46) and with the help of Theorems 2 and 5, we can state the following integration by parts theorem for the Caputo case.

Proposition 4 (Integration by parts for the Caputo type derivative ${ }_{a}^{\mathrm{ABC}} D^{\alpha, \mu, \gamma}$ ) Let $0<\alpha<$ $1, \mu>0, \gamma \in \mathbb{N}$, then

$$
\begin{aligned}
& \text { - } \int_{a}^{b}\left({ }_{a}^{\mathrm{ABC}} D^{\alpha, \mu, \gamma} f\right)(t) g(t)=\int_{a}^{b} f(t)\left({ }^{\mathrm{ABR}} D_{b}^{\alpha, \mu, \gamma} g\right)(t)+\left.\frac{B(\alpha)}{1-\alpha} f(t)\left(\mathbf{E}_{\alpha, \mu, \frac{-\alpha}{1-\alpha}, b^{-}}^{\gamma}\right)(t)\right|_{a} ^{b} \text {. } \\
& \text { - } \int_{a}^{b}\left({ }^{\mathrm{ABC}} D_{b}^{\alpha} f\right)(t) g(t)=\int_{a}^{b} f(t)\left(_{a}^{\mathrm{ABR}} D^{\alpha, \mu, \gamma} g\right)(t)-\left.\frac{B(\alpha)}{1-\alpha} f(t)\left(\mathbf{E}_{\alpha, \mu, \frac{-\alpha}{1-\alpha}, a^{+}}^{\gamma} g\right)(t)\right|_{a} ^{b} \text {. }
\end{aligned}
$$




\section{The related fractional Euler-Lagrange equations}

Below we investigate the corresponding Euler-Lagrange equations for a Lagrangian possessing the left new Caputo derivative with three parameter Mittag-Leffler kernel.

Theorem 6 Let $0<\alpha \leq 1, \mu>0, \gamma \in \mathbb{N}$, and $a<b$ be real numbers. Suppose that the functional $J: C^{2}[0, b] \rightarrow \mathbb{R}$, namely

$$
J(f)=\int_{0}^{b} L\left(t, f(t),{ }_{a}^{\mathrm{ABC}} D^{\alpha, \mu, \gamma} f(t)\right) d t
$$

possesses a local extremum in $S=\left\{y \in C^{2}[a, b]: y(a)=A, y(b)=B\right\}$ at some $f \in S$, keeping in mind that $L:[a, b] \times \mathbb{R} \times \mathbb{R} \rightarrow \mathbb{R}$. Then

$$
\left[L_{1}(s)+{ }^{\mathrm{ABR}} D_{b}^{\alpha, \mu, \gamma} L_{2}(s)\right]=0, \quad \text { for all } s \in[a, b],
$$

where $L_{1}(s)=\frac{\partial L}{\partial f}(s)$ and $L_{2}(s)=\frac{\partial L}{\partial_{a}^{\mathrm{ABC}} D^{\alpha, \mu, \gamma f}}(s)$.

Proof Let us suppose that $J$ has local maximum in $S$ at $f$. Thus, there exists $\epsilon>0$ fulfilling $J(\widehat{f})-J(f) \leq 0$ for all $\widehat{f} \in S$ with $\|\widehat{f}-f\|=\sup _{t \in[a, b]}|\widehat{f}(t)-f(t)|<\epsilon$. For any $\widehat{f} \in S$, there is $\eta \in H=\left\{y \in C^{2}[a, b], y(a)=y(b)=0\right\}$ fulfilling $\widehat{f}=f+\epsilon \eta$. As a result, the $\epsilon$-Taylor theorem provides the following:

$$
\begin{aligned}
L(t, f, \widehat{f}) & =L\left(t, f+\epsilon \eta,_{a}^{\mathrm{ABC}} D^{\alpha, \mu, \gamma} f+\epsilon_{a}^{\mathrm{ABC}} D^{\alpha, \mu, \gamma} \eta\right) \\
& =L\left(t, f,{ }_{a}^{\mathrm{ABC}} D^{\alpha, \mu, \gamma} f\right)+\epsilon\left[\eta L_{1}+{ }_{a}^{\mathrm{ABC}} D^{\alpha, \mu, \gamma} \eta L_{2}\right]+O\left(\epsilon^{2}\right) .
\end{aligned}
$$

Then

$$
\begin{aligned}
J(\widehat{f})-J(f) & =\int_{a}^{b} L\left(t, \widehat{f}(t),{ }_{a}^{\mathrm{ABC}} D^{\alpha, \mu, \gamma} \widehat{f}(t)\right)-\int_{a}^{b} L\left(t, f(t),{ }_{a}^{\mathrm{ABC}} D^{\alpha, \mu, \gamma} f(t)\right) \\
& =\epsilon \int_{a}^{b}\left[\eta(t) L_{1}(t)+\left({ }_{a}^{\mathrm{ABC}} D^{\alpha, \mu, \gamma} \eta\right)(t) L_{2}(t)\right]+O\left(\epsilon^{2}\right) .
\end{aligned}
$$

Here $\left.\delta J(\eta, y)=\int_{a}^{b}\left[\eta(t) L_{1}(t)+{ }_{0}^{\mathrm{ABC}} D^{\alpha, \mu, \gamma} \eta\right)(t) L_{2}(t)\right] d t$ represents the first variation of $J$.

After some calculations and using the integration by parts formula in Proposition 4, we get

$$
\delta J(\eta, y)=\int_{a}^{b} \eta(s)\left[L_{1}(s)+{ }^{\mathrm{ABR}} D_{b}^{\alpha, \mu, \gamma} L_{2}(s)\right]+\left.\eta(t) \frac{B(\alpha)}{1-\alpha}\left(\mathbf{E}_{\alpha, \mu, \frac{-\alpha}{1-\alpha}, b^{-}}^{\gamma} L_{2}\right)(t)\right|_{a} ^{b}=0
$$

for all $\eta \in H$, and hence the result follows by the fundamental lemma of calculus of variation.

Here, the quantity $\left.\left(\mathbf{E}_{\alpha, \mu, \frac{-\alpha}{1-\alpha}, b^{-}}^{\gamma} L_{2}\right)(t)\right|_{a} ^{b}=0$ above is named the natural boundary condition.

For the special case when the Lagrangian depends on the right Caputo derivative, we have the following. 
Theorem 7 Let us consider $0<\alpha \leq 1, \mu>0, \gamma \in \mathbb{N}$, and $a_{1}<b_{1}$ be real numbers. Assume that the functional $J: C^{2}\left[a_{1}, b_{1}\right] \rightarrow \mathbb{R}$ of the form

$$
J(f)=\int_{a_{1}}^{b_{1}} L\left(t, f(t),{ }^{\mathrm{ABC}} D_{b_{1}}^{\alpha, \mu, \gamma} f(t)\right) d t
$$

possesses a local extremum in $S=\left\{y \in C^{2}\left[a_{1}, b_{1}\right]: y\left(a_{1}\right)=A, y\left(b_{1}\right)=B\right\}$ at some $f \in S$, where $L:\left[a_{1}, b_{1}\right] \times \mathbb{R} \times \mathbb{R} \rightarrow \mathbb{R}$. Then

$$
\left[L_{1}(s)+{ }_{a_{1}}^{\mathrm{ABR}} D^{\alpha} L_{2}(s)\right]=0 \quad \text { for all } s \in\left[a_{1}, b_{1}\right],
$$

where $L_{1}(s)=\frac{\partial L}{\partial f}(s)$ and $L_{2}(s)=\frac{\partial L}{\partial^{\mathrm{ABC}} D_{b 1} 1^{\alpha, \mu, \gamma f}}(s)$.

Proof The proof looks similar to Theorem 6.

Example 1 We analyze the following example of physical interest by Theorem 6. Let us consider $J(y)=\int_{a}^{b}\left[\frac{1}{2}\left({ }_{a}^{\mathrm{ABC}} D^{\alpha, \mu, \gamma} y(t)\right)^{2}-V(y(t))\right]$, where $0<\alpha<1, \mu>0, \gamma \in \mathbb{N}$ and with $y(a)$, $y(b)$ are assigned or with the natural boundary condition

$$
\left.\left(\mathbf{E}_{\alpha, \mu, \frac{-\alpha}{1-\alpha}, b^{-} a}^{\mathrm{ABC}} D^{\alpha, \mu, \gamma} y(t)\right)(t)\right|_{a} ^{b}=0 .
$$

As a result, the related Euler-Lagrange equation becomes

$$
\left({ }^{\mathrm{ABR}} D_{b}^{\alpha, \mu, \gamma} \underset{a}{\mathrm{ABC}} D^{\alpha, \mu, \gamma} y\right)(s)-\frac{d V}{d y}(s)=0 \quad \text { for all } s \in[a, b] .
$$

We noticed that the Euler-Lagrange equations consist of composition of left and right type fractional derivatives with three parameter ML-kernels (see [4] for more details).

- The free particle case corresponds to $V \equiv 0$. In this case we get

$$
\left({ }^{\mathrm{ABR}} D_{b}^{\alpha, \mu, \gamma} \underset{a}{\mathrm{ABC}} D^{\alpha, \mu, \gamma} y\right)(t)=0 .
$$

By applying ${ }^{\mathrm{AB}} I_{b}^{\alpha, \mu, \gamma}$ to both sides, we conclude that

$$
\left(\begin{array}{l}
\mathrm{ABC} \\
a
\end{array} D^{\alpha, \mu, \gamma} y\right)(t)=0 .
$$

Then, by Remark 3, it implies that the solution is written as

$$
y(t)=c_{1}+G_{\gamma}(x-a)=c_{1}+\frac{1-\alpha}{B(\alpha)} E_{\alpha, \nu}^{-\gamma}(\lambda, x-a), \quad v \rightarrow 1-\mu,
$$

and hence using $y(a)=A$ we obtain

$$
y(t)=y(a)+G_{\gamma}(x-a) .
$$

For example,

$$
\begin{aligned}
G_{1}(x-a) & =\frac{1-\alpha}{B(\alpha)}\left[\frac{(x-a)^{-\mu}}{\Gamma(1-\mu)}-\lambda \frac{(x-a)^{\alpha-\mu}}{\Gamma(\alpha+1-\mu)}\right] \\
& =\frac{1-\alpha}{B(\alpha)} \frac{(x-a)^{-\mu}}{\Gamma(1-\mu)}+\frac{\alpha}{B(\alpha)} \frac{(x-a)^{\alpha-\mu}}{\Gamma(\alpha+1-\mu)} .
\end{aligned}
$$


Notice that $\mu \rightarrow 1^{-}$and $a=0$ will result in the function $g(x)=\frac{\alpha x^{\alpha-1}}{B(\alpha) \Gamma(\alpha)}$ proposed in [17]. We remark here that as alpha goes to one, we get the classical case. For $\gamma=2$, we have by $(-2)_{0}=1,(-2)_{1}=-2,(-2)_{2}=2$

$$
\begin{aligned}
G_{2}(x-a)= & \frac{1-\alpha}{B(\alpha)}\left[\frac{(x-a)^{-\mu}}{\Gamma(1-\mu)}-2 \lambda \frac{(x-a)^{\alpha-\mu}}{\Gamma(\alpha+1-\mu)}\right. \\
& \left.+2 \lambda^{2} \frac{(x-a)^{2 \alpha-\mu}}{\Gamma(2 \alpha+1-\mu)}\right] \\
= & \frac{1-\alpha}{B(\alpha)} \frac{(x-a)^{-\mu}}{\Gamma(1-\mu)}+2 \frac{\alpha}{B(\alpha)} \frac{(x-a)^{\alpha-\mu}}{\Gamma(\alpha+1-\mu)} \\
& +\frac{2 \alpha^{2}}{B(\alpha)(1-\alpha)} \frac{(x-a)^{2 \alpha-\mu}}{\Gamma(2 \alpha+1-\mu)} .
\end{aligned}
$$

Hence, $G_{\gamma}(x-a)$ is singular at $\alpha=1$ for $\gamma=2,3,4, \ldots$.

- Considering $V(y)=c y^{2} / 2$, the expression of the fractional Euler-Lagrange equation is written as $\left({ }^{\mathrm{ABR}} D_{b a}^{\alpha \mathrm{ABC}} D^{\alpha, \mu, \gamma} y\right)(t)=c y(t)$. Then, applying ${ }^{\mathrm{AB}} I_{b}^{\alpha, \mu, \gamma}$ and ${ }_{a}^{\mathrm{AB}} I^{\alpha, \mu, \gamma}$

respectively together with use of Theorem 3 , we obtain

$$
y(t)=y(a)+c\left({ }_{a}^{\mathrm{AB}} I^{\alpha, \mu, \gamma \mathrm{AB}} I_{b}^{\alpha, \mu, \gamma} y\right)(t)
$$

which contains, when $\alpha$ tends to 1 , the classical case.

\section{Conclusions}

The fractional derivatives studied in $[15,17]$ are of interest for real world problems since they contain nonsingular Mittag-Leffler kernels and their corresponding fractional integrals are expressed by mean of the classical Riemann fractional integrals. They also obey the action of $Q$-operator in studying the left and right fractional operators. In this article we have generalized the results obtained in $[15,17]$ by defining fractional derivatives with Mittag-Leffler kernels of three parameters $0<\alpha<1, \mu>0, \gamma \in \mathbb{R}$ and then obtained their corresponding fractional integrals when the function is regular at $a$ (the non-regular case is still open). Such kernels might be singular depending on the value of $\mu$. For such fractional derivatives either in Riemann (ABR) or Caputo (ABC) sense, we proved integration by parts when $\gamma \in \mathbb{N}$ since we were able just to calculate the corresponding fractional integrals by Laplace transforms. We have applied our integration by parts on a fractional variational problem with Lagrangian containing left or right ABC-derivatives, after which we analyzed a detailed example of physical interest, where nonconstant solutions expressed by means of $G_{\gamma}(x-a), \gamma=1,2, \ldots$, have been obtained for zero potential Lagrangian. The action of the corresponding proposed left and right fractional integrals on left and right $\mathrm{ABC}$ derivatives has been studied with the surprising conclusion that this action does not depend on the three parameters of the Mittag-Leffler kernel (see Theorem 3). This action is useful to solve fractional dynamical systems with $\mathrm{ABC}$ derivatives. We have related the ABR and ABC derivatives for arbitrary $\gamma$ (see Theorem 2). All the results obtained in [15, 17] can be recovered by setting $\gamma=\mu=1$. 
Availability of data and materials

Data sharing not applicable to this article as no datasets were generated or analyzed during the current study.

\section{Competing interests}

The authors declare that they have no competing interests.

\section{Authors' contributions}

All authors read and approved the final manuscript.

\section{Author details}

'Department of Mathematics and General Sciences, Prince Sultan University, Riyadh, Saudi Arabia. ${ }^{2}$ Department of Mathematics, Cankaya University, Ankara, Turkey. ${ }^{3}$ Institute of Space Sciences, Magurele-Bucharest, Romania.

\section{Publisher's Note}

Springer Nature remains neutral with regard to jurisdictional claims in published maps and institutional affiliations.

Received: 27 July 2018 Accepted: 3 December 2018 Published online: 19 December 2018

\section{References}

1. Podlubny, I.: Fractional Differential Equations. Academic Press, San Diego (1999)

2. Samko, S.G., Kilbas, A.A., Marichev, O.I.: Fractional Integrals and Derivatives: Theory and Applications. Gordon and Breach, Yverdon (1993)

3. Kilbas, A., Srivastava, H.M., Trujillo, J.J.: Theory and Application of Fractional Differential Equations. Mathematics Studies, vol. 204. North-Holland, Amsterdam (2006)

4. Abdeljawad, T., Baleanu, D., Jarad, F.: Existence and uniqueness theorem for a class of delay differential equations with left and right Caputo fractional derivatives. J. Math. Phys. 49, 083507 (2008)

5. Magin, R.L.: Fractional Calculus in Bioengineering. Begell House Publishers (2006)

6. Baleanu, D., Diethelm, K., Scalas, E., Trujillo, J.J.: Fractional Calculus Models and Numerical Methods, Series on Complexity, Nonlinearity and Chaos. World Scientific, Singapore (2012)

7. Hilfer, R.: Applications of Fractional Calculus in Physics. World Scientific, Singapore (2000)

8. Bagley, R.L., Torvik, P.J.: A generalized derivative model for elastomer damper. Shock Vib. Bull. 49, 135-143 (1979)

9. Yang, X.Y., Gao, F., Srivastava, H.M.: A new computational approach for solving nonlinear local fractional PDEs. J. Comput. Appl. Math. 339(339), 285-296 (2018)

10. Yang, X.J., Tenreiro Machado, J.A., Srivastava, H.M.: A new fractional derivative without singular kernel: application to the modelling of the steady heat flow. Therm. Sci. 20(2), 753-756 (2016)

11. Yang, X.J., Gao, F., Tenreiro Machado, J.A., Baleanu, D.: A new fractional derivative involving the normalized sinc function without singular kernel. Eur. Phys. J. Spec. Top. 226(16-18), 3567-3575 (2017)

12. Yang, X.J.: New rheological problems involving general fractional derivatives with nonsingular power-law kernels. Proc. Rom. Acad., Ser. A: Math. Phys. Tech. Sci. Inf. Sci. 19(1), 45-52 (2018)

13. Yang, X.Y., Tenreiro Machado, J.A., Baleanu, D.: Anomalous diffusion models with general fractional derivatives within the kernels of the extended Mittag-Leffler type functions. Rom. Rep. Phys. 69(4), 115 (2017)

14. Caputo, M., Fabrizio, M.: A new definition of fractional derivative without singular kernel. Prog. Fract. Differ. Appl. 1(2), 73-85 (2015)

15. Atangana, A., Baleanu, D.: New fractional derivative with non-local and non-singular kernel. Therm. Sci. 20(2), 757-763 (2016)

16. Abdeljawad, T., Baleanu, D.: Discrete fractional differences with nonsingular discrete Mittag-Leffler kernels. Adv. Differ. Equ. 2016, 232 (2016). https://doi.org/10.1186/s13662-016-0949-5

17. Abdeljawad, T., Baleanu, D.: Integration by parts and its applications of a new nonlocal fractional derivative with Mittag-Leffler nonsingular kernel. J. Nonlinear Sci. Appl. 10(3), 1098-1107 (2017)

18. Abdeljawad, T., Baleanu, D.: On fractional derivatives with exponential kernel and their discrete versions. Rep. Math. Phys. 80(1), 11-27 (2017)

19. Abdeljawad, T., Al-Mdallal, Q.M.: Discrete Mittag-Leffler kernel type fractional difference initial value problems and Gronwall's inequality. J. Comput. Appl. Math. 339, 218-230 (2018). https://doi.org/10.1016/j.cam.2017.10.021

20. Abdeljawad, T.: Fractional operators with exponential kernels and a Lyapunov type inequality. Adv. Differ. Equ. 2017, 313 (2017)

21. Abdeljawad, T.: A Lyapunov type inequality for fractional operators with nonsingular Mittag-Leffler kernel. J. Inequal. Appl. 2017, $130(2017)$

22. Abdeljawad, T., Al-Mdallal, Q.M., Hajji, M.A.: Arbitrary order fractional difference operators with discrete exponential kernels and applications. Discrete Dyn. Nat. Soc. 2017, Article ID 4149320 (2017)

23. Abdeljawad, T., Madjidi, F.: Lyapunov-type inequalities for fractional difference operators with discrete Mittag-Leffler kernel of order $2<\alpha<5 / 2$. Eur. Phys. J. Spec. Top. 226(16-18), 3355-3368 (2017)

24. Abdeljawad, T., Baleanu, D.: Monotonicity analysis of a nabla discrete fractional operator with discrete Mittag-Leffler kernel. Chaos Solitons Fractals 102 106-110 (2017)

25. Abdeljawad, T., Baleanu, D.: Monotonicity results for fractional difference operators with discrete exponential kernels. Adv. Differ. Equ. 2017, 78 (2017). https://doi.org/10.1186/s13662-017-1126-1

26. Baleanu, D., Jihad, A., Petras, l.: Numerical solutions of the fractional Euler-Lagrange's equations of a thin elastica model. Nonlinear Dyn. 81(1-2), 97-102 (2015)

27. Bouzid, N., Merad, M., Baleanu, D.: On fractional Duffin-Kemmer-Petiau equation. Few-Body Syst. 57(4), 265-273 (2016)

28. Agila, A., Baleanu, D., Eid, R., Irfanoglu, B.: Applications of the extended fractional Euler-Lagrange equations model to freely oscillating dynamical systems. Rom. J. Phys. 61(3-4), 350-359 (2016) 
29. Baleanu, D., Abdeljawad, T., Jarad, F.: Fractional variational principles with delay. J. Phys. A, Math. Theor. 41(31), 315403 (2008)

30. Jarad, F., Abdeljawad, T., Baleanu, D.: On Riesz-Caputo formulation for sequential fractional variational principles. Abstr. Appl. Anal. 2012, Article ID 890396 (2012)

31. Jarad, F., Abdeljawad, T., Baleanu, D.: Fractional variational optimal control problems with delayed arguments. Nonlinear Dyn. 62(3), 609-614 (2010)

32. Jarad, F., Abdeljawad, T., Baleanu, D.: Fractional variational principles with delay within Caputo derivatives. Rep. Math. Phys. 65, 17-28 (2010)

33. Jarad, F., Abdeljawad, T., Baleanu, D.: Higher order variational optimal control problems with delayed arguments. Appl. Math. Comput. 218(18), 9234-9240 (2012)

34. Prabhakar, T.R.: A singular integral equation with a generalized Mittag-Leffler function in the kernel. Yokohama Math. J. 19, 7-15 (1971)

35. Kilbas, A.A., Saigo, M., Saxena, K.: Generalized Mittag-Leffler function and generalized fractional calculus operators. Integral Transforms Spec. Funct. 15(1), 31-49 (2004)

36. Abdeljawad, T:: On delta and nabla Caputo fractional differences and dual identities. Discrete Dyn. Nat. Soc. 201, Article ID 406910 (2013)

37. Abdeljawad, T.: Dual identities in fractional difference calculus within Riemann. Adv. Differ. Equ. 2013, 36 (2013)

\section{Submit your manuscript to a SpringerOpen ${ }^{\circ}$ journal and benefit from:}

- Convenient online submission

- Rigorous peer review

- Open access: articles freely available online

- High visibility within the field

- Retaining the copyright to your article

Submit your next manuscript at $\gg$ springeropen.com 